\title{
Studener-Kuras, Regina
}

\section{"Dass ich Lehrerin bin, das habe ich auf eine recht eigene Art ganz vergessen gehabt!". Lehren und Lernen im Kontext von Flucht und Neubeginn \\ Kremsner, Gertraud [Hrsg.]; Proyer, Michelle [Hrsg.]; Biewer, Gottfried [Hrsg.]: Inklusion von Lehrkräften nach der Flucht. Über universitäre Ausbildung zum beruflichen Wiedereinstieg. Bad Heilbrunn : Verlag Julius Klinkhardt 2020, S. 100-107}

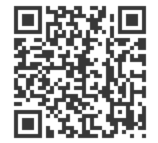

Quellenangabe/ Reference:

Studener-Kuras, Regina: "Dass ich Lehrerin bin, das habe ich auf eine recht eigene Art ganz vergessen gehabt!". Lehren und Lernen im Kontext von Flucht und Neubeginn - In: Kremsner, Gertraud [Hrsg.]; Proyer, Michelle [Hrsg.]; Biewer, Gottfried [Hrsg.]: Inklusion von Lehrkräften nach der Flucht. Über universitäre Ausbildung zum beruflichen Wiedereinstieg. Bad Heilbrunn : Verlag Julius Klinkhardt 2020, S. 100-107 - URN: urn:nbn:de:0111-pedocs-189120 - DOI: 10.25656/01:18912

https://nbn-resolving.org/urn:nbn:de:0111-pedocs-189120

https://doi.org/10.25656/01:18912

in Kooperation mit / in cooperation with:

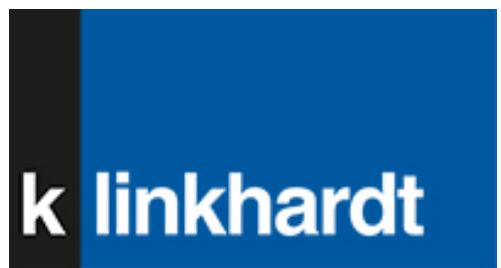

http://www.klinkhardt.de

\section{Nutzungsbedingungen}

Dieses Dokument steht unter folgender Creative Commons-Lizenz: http://creativecommons.org/licenses/by-nc-sa/4.0/deed.de - Sie dürfen das Werk bzw. den Inhalt unter folgenden Bedingungen vervielfältigen, verbreiten und öffentlich zugänglich machen sowie Abwandlungen und Bearbeitungen des Werkes bzw. Inhaltes anfertigen: Sie müssen den Namen des Autors/Rechteinhabers in der von ihm festgelegten Weise nennen. Dieses Werk bzw. der Inhalt darf nicht für kommerzielle Zwecke verwendet werden. Die neu entstandenen Werke bzw. Inhalte dürfen nur unter Verwendung von Lizenzbedingungen weitergegeben werden, die mit denen dieses Lizenzvertrages identisch oder vergleichbar sind.

Mit der Verwendung dieses Dokuments erkennen Sie die Nutzungsbedingungen an.

\section{Terms of use}

This document is published under following Creative Commons-License: http://creativecommons.org/licenses/by-nc-sa/4.0/deed.en - You may copy, distribute and transmit, adapt or exhibit the work in the public and alter, transform or change this work as long as you attribute the work in the manner specified by the author or licensor. You are not allowed to make commercial use of the work. If you alter, transform, or change this work in any way, you may distribute the resulting work only under this or a comparable license.

By using this particular document, you accept the above-stated conditions of use.

\section{Kontakt / Contact:}

peDOcs

DIPF | Leibniz-Institut für Bildungsforschung und Bildungsinformation

Informationszentrum (IZ) Bildung

E-Mail: pedocs@dipf.de

Internet: www.pedocs.de

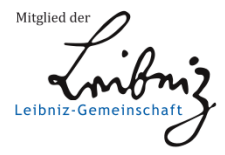




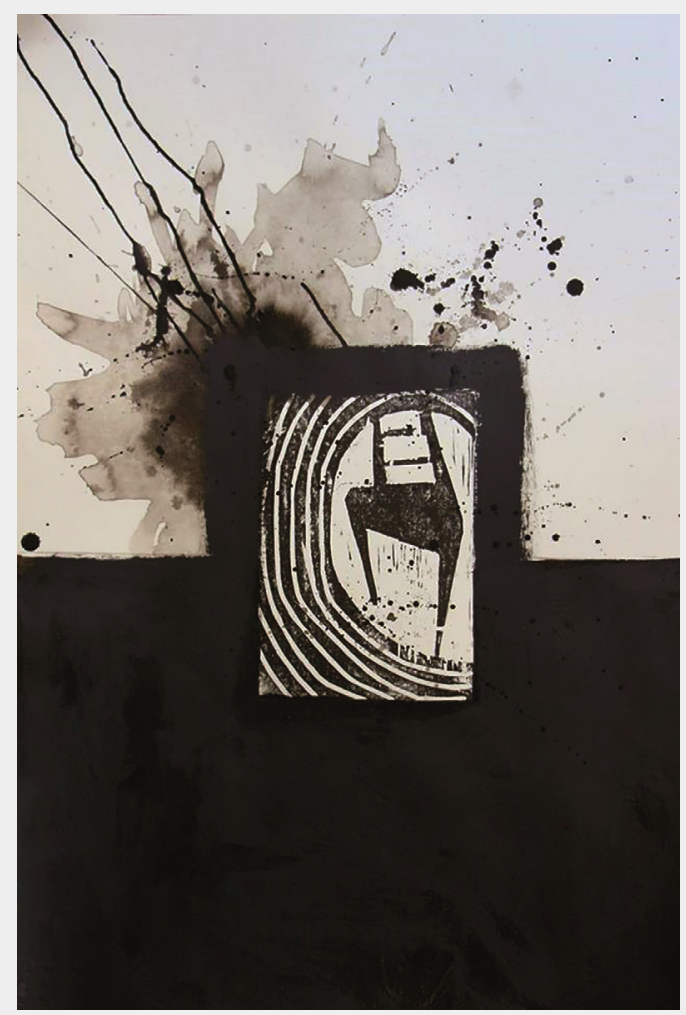

Gertraud Kremsner Michelle Proyer

Gottfried Biewer

(Hrsg.)

Inklusion von Lehrkräften nach der Flucht

Über universitäre Ausbildung zum beruflichen Wiedereinstieg 


\section{Gertraud Kremsner Michelle Proyer Gottfried Biewer (Hrsg.)}

\section{Inklusion von Lehrkräften nach der Flucht}

Über universitäre Ausbildung zum beruflichen Wiedereinstieg 
„Allen Personen gewidmet, die geflïchtet sind, sich derzeit auf der Flucht befinden oder noch flïchten werden. "

Die Publikation des Buches und die Open Access-Veröffentlichung wurde vom Zentrum für Lehrer*innenbildung und vom Postgraduate Center der Universität Wien bezuschusst.

Dieser Titel wurde in das Programm des Verlages mittels eines Peer-Review-Verfahrens aufgenommen. Für weitere Informationen siehe www.klinkhardt.de.

Bibliografische Information der Deutschen Nationalbibliothek Die Deutsche Nationalbibliothek verzeichnet diese Publikation in der Deutschen Nationalbibliografie; detaillierte bibliografische Daten sind im Internet abrufbar über http://dnb.d-nb.de.

2020.ig. (C) by Julius Klinkhardt.

Satz: Tina Obermayr, Wien

Abbildung Umschlagseite 1: Marwa Sarah (Österreich/Syrien) - Black Hole

The painful fact for a refugee or a foreigner is that you will be always looking for a place to belong to, and you will never find it again you will become a foreigner everywhere you go, slowly you will change and do not fit anywhere. and there will always be a black hole. black hole.

Druck und Bindung: AZ Druck und Datentechnik, Kempten.

Printed in Germany 2020.

Gedruckt auf chlorfrei gebleichtem alterungsbeständigem Papier.

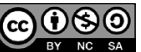

Die Publikation (mit Ausnahme aller Fotos, Grafiken und Abbildungen) ist veröffentlicht unter der Creative Commons-Lizenz: CC BY-NC-SA 4.0 International https://creativecommons.org/licenses/by-nc-sa/4.0/ 


\section{Inhaltsverzeichnis}

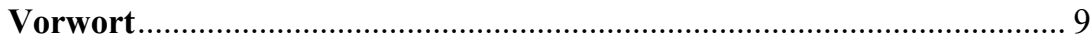

\section{Darstellungen und Forschungen zum Zertifikatskurs}

Gertraud Kremsner, Michelle Proyer und Tina Obermayr

Die Ausgangslage und die Einrichtung des Zertifikatskurses

„Bildungswissenschaftliche Grundlagen für

Lehrkräfte mit Fluchthintergrund“.

Gertraud Kremsner, Michelle Proyer und Alexander Schmölz mit Unterstützung von Helena Deiß, Lisa-Katharina Möhlen, Sarah Hofmann, Marwa Sarah und Tina Obermayr

Das Forschungsprojekt „Qualifizierung von Lehrkräften mit Fluchthintergrund“" 46

\section{Reflexion der Kursinhalte aus Sicht der Lehrenden}

Ines M. Breinbauer

Wie man in die Pädagogik einführen und dabei selber viel lernen kann!

Bildungswissenschaftliche Grundlagen in der Lehrer*innenbildung für Lehrpersonen mit Fluchthintergrund.

Regina Studener-Kuras

„Dass ich Lehrerin bin, das habe ich auf eine recht eigene Art ganz vergessen gehabt!" Lehren und Lernen im Kontext von Flucht und Neubeginn. 100

Michelle Proyer

,Ich male denen die Perspektive“ - Von Praxiserfahrungen zwischen ,bei uns' und ,bei euch', über das Erlernen von ,Reflexion` und hin zu einem ,Ankommen` im österreichischen Schulsystem. 


\section{6 | Inhaltsverzeichnis}

Neda Forghani-Arani

Lived Experience of Teaching Displaced Teachers:

A Postcolonial Reading of Positions, Voices and Representations

Gottfried Biewer

„Inklusive Pädagogik und Vielfalt"

für Lehrkräfte mit Fluchthintergrund

Sabine Krause

Schulforschung und Unterrichtspraxis. Bewegungen zwischen

wissenschaftlicher Abstraktion und „Praxisrelevanz“

Raphael Zahnd und Gertraud Kremsner

Zur vertieften Auseinandersetzung mit Heterogenität in Schulkontexten... 134

Michael Doblmair und Michelle Proyer

Am Ende steht (wieder) die Reflexion

\section{Herausforderungen und Synergien}

Michelle Proyer, Gertraud Kremsner, Gottfried Biewer und Camilla Pellech

Herausforderungen und Synergien aus universitärer Perspektive

Linda Kreuter, Helena Deiß, Lisa-Katharina Möhlen, Kamal Alyouzbashi, Saad Chatto, Sahar Hashemi, Nizar Mousa, Doha Tahlawi, Ahmed Zeki Al Hamid und Jomard Rasul „Werden Träume wahr?“ - Reflexionen der Kursteilnehmer*innen

Marie-Claire Sowinetz

„Nehmen wir das Gute von uns und das Gute von euch so werden wir alle besser." Ein persönlicher Rückblick

auf den Beginn des Zertifikatskurses für geflüchtete Lehrer*innen 166

Katharina Resch

Vier Strategien zur Entwicklung von universitären

Weiterbildungsprogrammen im Bereich Flucht und Migration 
Renate Faistauer, Thomas Laimer und Nicola Kraml

Beitrag zu einer nachhaltigen Sprachförderung für Lehrende

mit Fluchthintergrund - Synergien in der Ausbildung schaffen

und Empowerment bei den Teilnehmer*innen ermöglichen.

Karoline Gerwisch, Denise Strehn, Nicolas Kieffer

und Michelle Proyer

Reflexion der Kurspraktika -

Perspektiven der Mentor*innen und Mentees 184

\section{Internationale Perspektiven}

Annika Käck

Migrant teachers in Swedish teacher education and their

re-entry as professionals

Susanna Malm

Bridging Programmes for Migrant Teachers

and Preschool Teachers in Sweden.

Henrike Terhart, Ariane Elshof und Susanne Preuschoff

Programm für geflüchtete Lehrkräfte an der Universität zu Köln.

Kristina Purrmann, Renate Schüssler, Christina Siebert-Husmann

und Marie Vanderbeke

„Wir haben so lange auf eine Chance gewartet“ -

Potentiale und Herausforderungen des Qualifizierungsprogrammes

Lehrkräfte Plus für geflüchtete Lehrkräfte

Katja Kansteiner, Roswitha Klepser, Sarah Lukas,

Kristin Rheinwald und Tim Kaiser

Integration geflüchteter Lehrkräfte in die Lehrer*innenausbildung in Baden-Württemberg - das IGEL-Programm.

Abschlussbemerkungen 


\section{„Dass ich Lehrerin bin, das habe ich auf eine recht eigene Art ganz vergessen gehabt!'‘ Lehren und Lernen im Kontext von Flucht und Neubeginn}

Überall geht ein früheres Ahnen dem späteren Wissen voraus (Humboldt).

\section{Konzeption, theoretischer Hintergrund und Zielsetzung des Moduls Entwicklung und Bildung}

Die inhaltlichen Schwerpunktsetzungen dieses Moduls lagen in der Auseinandersetzung mit kindlichen Entwicklungsprozessen im Kontext von schulischem Lernen in der Sekundarstufe. Im Fokus standen dabei sowohl die Vermittlung und das Kennenlernen theoretischer Konzepte kindlicher Entwicklung als auch die Frage nach der Bedeutung von Emotionen und Beziehungserfahrungen für Lern- und Entwicklungsprozesse. Inhaltliche Querschnittsthemen waren dabei spezifische Überlegungen zur Auseinandersetzung mit Bedingungen des Lehrens und Lernens aus bildungswissenschaftlicher und entwicklungspsychologischer Sicht mit besonderem Fokus auf Schüler*innen mit Flucht- und Migrationserfahrung.

Ich unterrichte im Themenfeld Entwicklungspsychologie nun schon seit geraumer Zeit, meist mit teilflexibler inhaltlicher, didaktischer und struktureller Ausrichtung, sodass sowohl die Zielsetzung des spezifischen Ausbildungslehrganges als auch die Individualität der jeweiligen Fortbildungsgruppe ausreichend Platz finden kann. Der ,kleinste gemeinsame konzeptionelle Nenner' liegt für mich dabei auf dreierlei Gesichtspunkten:

- Einer inhaltlichen Positionierung von Entwicklungspsychologie, die an aktuellen Forschungsergebnissen angelehnt und an psychoanalytischen Theorien zur kindlichen Entwicklung orientiert ist,

- einem didaktischen Dialog, in dem sich referierendes Vortragen, Kleingruppenarbeit und die Auseinandersetzung mit Texten und Praxisbeispielen abwechseln und

- biographischem Nachdenken der Teilnehmer*innen über eigene Lernund Lehrerfahrungen und damit verbundenen Emotionen und Erinnerungen. 
Diese strukturellen Elemente bilden die tragenden Säulen meines Lehrkonzepts und bedingen im günstigsten Fall ein aufeinander bezogenes Verstehen von Theorie, Forschung und Praxis, das die vielschichtige Bedeutung psychodynamischen Geschehens für kindliche Lern- und Leistungsprozesse transparenter und für die pädagogische Praxis zugänglicher machen soll.

Ergänzend dazu sind meiner Erfahrung nach noch zwei weitere Aspekte für das Gelingen von Lehr- und Lernprozessen von hoher Relevanz:

- In Anlehnung an Salzberger-Wittenberg (1997) das Wahrnehmen und Einbeziehen jener Gefühle, die bei Lehrenden und Studierenden gerade zu Beginn des Unterrichtens spürbar sind und die das Zustandekommen des gemeinsamen Arbeitsbündnisses wesentlich beeinflussen;

- die Bedeutung von Beziehungserfahrungen und dem damit verbundenen Erleben von sich und der Welt, die u.a. auch für die Aufnahme von sowohl kognitiven als auch sozialen Lerninhalten entscheidend sind.

Ein kurzer Ausschnitt aus einer Unterrichtssequenz gibt im Folgenden Einblick in den gemeinsamen Lehr- und Lernprozess im Modul und zeigt, wie dicht theoretische Überlegungen, Praxiserfahrungen und Emotionales auch hier im Zertifikatskurs miteinander verwoben waren.

\section{1 Über das Beginnen, verborgene Ängste und die Notwendigkeit, im Unterricht über Emotionen zu sprechen}

Die englische Psychoanalytikerin Salzberger-Wittenberg forscht an der Tavistock Clinic in London seit vielen Jahren zur Bedeutung von emotionalem, psychodynamischem Geschehen im Kontext der Fortbildung von Pädagog*innen und weist in zahlreichen Publikationen darauf hin, dass gerade zu Beginn von Prozessen des Lehrens und Lernens eine Fülle an bewussten und unbewussten Gefühlen sowohl auf Seite der Lehrperson als auch auf jener der Schüler*innen spürbar sind. Diese werden in Übertragungs- und Gegenübertragungsprozessen deutlich und können zum Verstehen psychodynamischen Geschehens in Lernprozessen hilfreich sein. „Es gehört zum Wesen des Beginnens, dass wir den Weg vor uns nicht kennen und zwischen erregtem Staunen und banger Spannung schwanken, wenn wir ihn betreten" (ebd., 19). „Zweifel und Befürchtungen“, so Salzberger-Wittenberg, sind „ein wesentlicher Bestandteil des Beginnens" (ebd.).

So begann ich auf theoretischer Ebene erste Überlegungen über den Zusammenhang von psychodynamischem Geschehen und Lernprozessen (vgl. Gerspach 2018) im Unterricht vorzustellen, um in der nächstfolgenden Einheit mit den Teilnehmer*innen dazu ins Gespräch zu kommen. 
Das Besprechen von Gefühlen im Unterricht - im Speziellen von Gefühlen der Lehrperson - war für die Teilnehmer*innen ungewohnt und das Nachdenken über emotionale Befindlichkeiten und Beziehungserfahrungen im Klassenraum recht neu. Alle Studierenden waren jedoch offen, sich auf diese Art des Nachdenkens und Lernens einzulassen.

Insbesondere als ich darauf aufmerksam machte, dass viele der Gefühle, die sie bei sich wahrnehmen, mit ziemlicher Sicherheit auch manche der Kinder, die sie aktuell in ihrem Praktikum begleiteten, beschäftigen und dass diese Emotionen somit Teile des Lernprozesses sind und hemmenden, aber auch förderlichen Einfluss haben können. Diese Form der Selbstreflexion eröffnet für uns die Möglichkeit, das Verhalten von Schüler*innen aus einer erweiterten Perspektive zu verstehen und beim Unterrichten darauf Rücksicht zu nehmen.

\section{2 Über die Erschütterung von Erinnerung, den Verlust von beruflichem Selbstverständnis und Erfahrungen von Flucht und Migration}

Ich plante in weiterer Folge einen Text von Rüdi (2001) zur Interaktion von Schüler*innen und ihren Lehrer*innen vorzustellen und ermunterte die Studierenden ihre Erfahrungen mit Schüler*innen, die sie als Professor*innen in den Gymnasien und Sekundarstufen ihrer Heimatländer gemacht hatten, zu erzählen.

Eingedenk meiner Erfahrungen aus anderen Lehrveranstaltungen mit diesem Arbeitsformat ging ich davon aus, dass dies den Studierenden als Arbeitsauftrag leicht fallen und zu anregenden Diskussionen führen würde - zumal die Teilnehmer*innen, so meine Annahme, dadurch auch die Gelegenheit hatten, ihre eigenen Lehrerfahrungen einzubringen, an Vertrautes anzuknüpfen und im Modul aus der ,Schüler*innenposition“ heraustreten zu können. Die Schilderung von Unterrichtserlebnissen aus dem Blickwinkel der Lehrer*innenrolle würde es ermöglichen - so meine didaktische Idee -, Lernund Lehrprozesse und damit verbundene Beziehungserfahrungen aus verschiedenen Perspektiven zu betrachten und differenziert zu verstehen. In unserem Modul verlief dies jedoch anders als erwartet: Die Studierenden beantworteten meine Frage und mein Ersuchen, aus ihrer Lehr-Praxis zu erzählen, wohl bemüht und engagiert, jedoch meist mit der Schilderung von Erfahrungen, die sie selbst als Schüler*innen mit ihren Lehrer*innen gemacht hatten.

Zaghaft, fast stockend, erzählten zwei Teilnehmerinnen von einzelnen Erlebnissen als Lehrerin, dies allerdings meist im Zusammenhang mit Aktivitäten 
bei Sportveranstaltungen. Die Mehrzahl der Studierenden berichtete jedoch, dass sie sich nicht mehr genau erinnern könnten, da dies schon lange her sei und sie viel besser wüssten, wie es ihnen als Schüler*innen ergangen war. Ich meldete den Teilnehmer*innen in diesem Zusammenhang rück, dass ich dies für ein interessantes Phänomen hielt, insbesondere, da ja allen klar war, dass erstens die Erinnerungen an die eigene Schulzeit noch länger zurücklägen und zweitens die Erinnerungen, die sie als Lehrer*innen gemacht hatten, an sich vorhanden sein müssten und diese Erfahrungen ja auch, nicht einfach verschwinden:

Ich erzählte den Teilnehmer*innen, dass ich schon des Öfteren während unserer gemeinsamen Arbeit im Modul - meist dann, wenn ich sie auf ihre Rolle als Lehrer*innen in ihren ,alten Schulen' ansprach - den Eindruck hatte, sie würden sich zurückziehen. Als ob sich eine stille unsichtbare Distanz aufbauen würde und die spürbare zugewandte Anwesenheit im Raum verblassen würde; als ob sich in diesen Momenten ein voller Unterrichtsraum mit Studierenden in einen leeren Raum mit Menschen verwandelte und wir mit diesen Fragen so etwas wie ein Tabuthema berührten. Als ob jemand plötzlich im Anwesend-Sein abwesend wird; in einem Bus sitzend am Reiseziel ankommt, ohne je auszusteigen.

In diesen Worten beschrieb ich die Bilder und Empfindungen, die in mir auftauchten, während die Teilnehmer*innen sehr präsent meinen Ausführungen zuhörten. Da rief plötzlich einer der Teilnehmer*innen laut nach vorne zu mir und andere ergänzten beherzt:

- „Ja sicher, so ist doch unsere Realität. Wir haben alles verloren! Auch unser Lehrer-Sein, unseren Beruf, unseren Status, unseren beruflichen Erfolg. Wir sind keine Lehrer mehr, keine richtigen! Wir sind jetzt Flüchtlinge und Migranten, viel mehr noch, als wir je Lehrer waren! Deshalb erinnern wir uns nicht."

- „Ich muss für meine Familie sorgen, Lehrer war ich in einem früheren Leben, jetzt muss ich Geld verdienen."

- „Die Lehrerin in mir, die von damals, die ist in Wahrheit eigentlich nie hier angekommen."

- ,Wir können vielleicht gar nicht mehr unterrichten, wir haben alles verlernt. Der Krieg hat uns zerstört. Und wir sind gestört! Das sagt man von uns! Flüchtlinge aus dem Krieg sind das immer: traumatisiert und gestört. Auch wir hier. So in diesem Zustand können wir wahrscheinlich gar nicht mehr unterrichten! “ 


\section{4 | Regina Studener-Kuras}

Ich war von diesen - wenn auch mit leicht humorigem Unterton - mir zugerufenen Bemerkungen zutiefst berührt und erschüttert. Vor dem Hintergrund dieser plötzlich herausgerufenen inneren Annahmen über sich und ihre Lebenssituation begann ich, auch meine Gegenübertragungsempfindungen noch einmal zu reflektieren und in Hinblick auf ihre mögliche Bedeutung im $\mathrm{Zu}-$ sammenhang mit dem Selbstverständnis und der Identität der Teilnehmer*innen zu überdenken. Dabei fand ich mich - wie bereits öfters in diesem Modul - neuerlich in einer Situation, in der ich nur recht wenig Wissen darüber hatte, wie bzw. in welcher Hinsicht ich den nächsten Unterrichtsschritt setzen musste, damit der Lernfortgang im Modul seine positive Fortsetzung beibehalten konnte.

\section{3 Über die Wiederentdeckung von Identität und Selbstverständnis als Lehrer*in im Verlauf von Entwicklungs- und Bildungsprozessen}

Mit diesen gemeinsamen Lehr- und Lernerfahrungen im Hintergrund gingen wir eine Woche später in den nächstfolgenden Modulblock, der - wie schon länger mit den Teilnehmer*innen besprochen - dem Thema von Trauma und Traumatisierung im Rahmen kindlicher Entwicklungsprozesse zugedacht war.

Ich referierte unter anderem Konzepte zur Genese und Diagnose von Traumafolgestörungen (vgl. Streeck-Fischer 2014; Bogyi 2006) bei Kindern und Jugendlichen und skizzierte Möglichkeiten traumapädagogischer Interventionen und Therapie nach Weiss (2014). Dabei besprach ich in Anlehnung an Terr (1995) die Unterscheidung in verschiedene Typen von Traumata und erläuterte dazu Praxisbeispiele aus dem Alltag meiner Familienberatungstätigkeit.

Ich verwies aber auch auf die Forschungsarbeiten von Ottomayer (2013), der in seinen Publikationen unter anderem darauf hinweist, dass das Erleben von sehr bedrohlichen Erfahrungen nicht immer und notwendigerweise mit Traumatisierungen einhergehen muss und beim Vorhandensein emotional ausreichend haltender Beziehungen sowie bestimmter innerer und äußerer Protektoren, eine gelingende Bewältigung und gesunde Verarbeitung auch von sehr angsterzeugenden Situationen möglich ist. Ich erläuterte, dass Erfahrungen von Krieg, Flucht und Migration also nicht per se zu Traumatisierung, seelischen Schädigungen und Erkrankungen führen müssen.

Während meines Vortrags wurde es tief still im Raum und die Präsenz und unmittelbare Aufmerksamkeit der Gruppe war deutlich zu spüren. Als ich in weiterer Folge begann, auf Publikationen Anna Freuds (1987) zu verweisen, die in ihren Studien zu Kriegskindern in London während des Zweiten Welt- 
kriegs zeigte, dass Kinder trotz Erfahrungen von Flucht und Verfolgung seelisch gesund aus dieser Zeit herausgegangen sind, begann unter den Teilnehmer*innen wuselige Unruhe aufzukommen. Ich wurde ersucht, diese Konzepte und Studien immer wieder aufs Neue zu erklären. Dabei merkte ich die starke emotionale Berührung, die bei manchen Mitgliedern der Gruppe aufkam, bis schließlich laut über jedes Gemurmel hinweg von einer Teilnehmerin die Frage an mich gestellt wurde, ob man somit aus wissenschaftlicher Sicht davon ausgehen könnte, ,, $\mathrm{d}$ ass sie, die Teilnehmer*innen dieser Zertifikatsausbildung, unter Umständen gar nicht alle traumatisiert sind“, „, sie keine Schädigungen davongetragen haben“ und unter Umständen „,nicht schwer gestört" sind. „Meinen Sie denn, es könnte sein, dass wir trotz Kriegserleben, Flucht und Asylverfahren genauso gesund sein könnten wie früher? Also genauso stark und seelisch intakt, wie wir das in unseren früheren Zeiten im Arbeitsleben zu Hause waren?"

Gespannt warteten alle auf meine Reaktion, zu der ich einerseits natürlich keine durchdachten Unterlagen in meiner Vorbereitung hatte und andererseits im selben Moment um die Verantwortung und das hohe Maß an Bedeutung wusste, das die gesamte Gruppe in meine Antwort legen würde: „Ich bin sicher“, sagte ich, ,,dass Sie Ihre Kraft, Ihre Zuversicht und Ihre Gesundheit durch die Erfahrungen, die Sie machen mussten, auch stärken konnten, auch wenn ich aber natürlich nicht in der Lage bin, diese Frage seriös und für alle Ihre individuellen Lebenssituationen entsprechend beantworten zu können. Ich kann aber aus meinen bisherigen Erfahrungen mit Ihnen in diesem Modul sagen, dass ich den Eindruck habe, dass Sie sich Ihre berufliche Gewordenheit und Kompetenz sowie Ihre Identität als lehrende und lernende Personen bewahrt haben und ich den Eindruck habe, dass Sie Ihr Selbstverständnis als Lehrer*innen bei sich wiedergefunden haben. Aus meiner Perspektive gesprochen, sogar dabei sind, dies zu stärken und zu bereichern!"

Ich bemerkte, mit welch aufmerksamer Stille die Teilnehmer*innen meinen Worten zugehört hatten, ohne jedoch genau einschätzen zu können, welche Reaktionen darauf folgen würden. Nach ein paar Momenten ergriff eine der Teilnehmerinnen die Hand ihrer Sitznachbarin und rief: „Ja! Stimmt, wir sind ja alle Lehrer, irgendwie habe ich das auf eine recht eigene Art ganz vergessen gehabt! " Unter den Teilnehmer*innen begann daraufhin eine Art gemeinsamer Gruppendialog, der aus einer Mischung von Ausrufen, lautformulierten Gedanken und leichtfühligem Herumscherzen bestand: ,, Jetzt habe ich mich als Ganzes wiedergefunden! „Ich BIN ja Lehrerin! Schon ganz lang! Das war weg, bei vollem Wissen untergetaucht! Wie in einer anderen Welt! " „,Jetzt erst sind wir sozusagen wieder ganz da!“ „, Ganz sind wir und nicht gestört! “, rief einer der Teilnehmer, mit einer Geste seiner Hände dabei nach 
oben streckend und einer emotionalen Mischung aus Stolz und Erleichterung, die mich an den Siegesjubel beim Gewinn einer Sportveranstaltung erinnerte. „Am besten wäre es jetzt, glaube ich“, wandte sich eine Teilnehmerin an mich, ,, wir könnten jetzt alles noch einmal lernen, denn ich habe das Gefühl, jetzt ist die Last weg und wir können uns viel mehr merken und besser verstehen!"

Der französische Psychologe Camilleri (1990) beschreibt Identitätsentwicklung als einen ,dynamischen Prozess, welcher lebenslang die verschiedenen Lebenserfahrungen des Individuums zu integrieren trachtet“ (ebd., 78). „In dem“, so die Schweizer Psychoanalytikerin Pedrina (1999), „verschiedene interaktionelle Situationen, verschiedene Aspekte der Identität anregen, die nicht bloß nebeneinander existieren, sondern sich zu einem mehr oder weniger kohärenten funktionellen Ganzen organisieren“ (ebd., 54).

Diese theoretischen Ausführungen und in weiterer Folge auch die Theorien Eriksons zu Identität und Lebenszyklus (1959) bildeten dann die weitere inhaltliche Basis, mit der wir uns in den weiteren Moduleinheiten beschäftigten und in denen sowohl die Studierenden als auch ich immer wieder überrascht und berührt waren, wie nahe Lehren und Lernen, das Nachdenken über Erfahrungen im Unterricht und eigenes biographisches Erleben in der Professionalisierung von Lehrer*innen beisammen liegen und einander bedingen.

Ich möchte auf diesem Weg den Teilnehmer*innen dieses Zertifikatskurses herzlich dafür danken, dass sie mir in diesem Fortbildungsmodul mit ihrem Engagement, ihrer Offenheit und ihrem Vertrauen, sowohl Einblick in das Unterrichten, Lehren und Lernen in anderen Ländern gegeben haben als auch ihre Erfahrung aus ihren langen Reisen der Flucht und Migration in den Lernprozess eingebracht haben und mir dadurch großartige Lehrer*innen waren.

„Die Wechselwirkung von äußerer, sich verändernder Realität und die Antwort der inneren - sich notwendigerweise ebenfalls verändernder - Realität beeinflusst alte und konstituiert neue innere Strukturen“" (Kothe-Meyer 1999, 74).

\section{Literatur}

Bogyi, G. (2006): Magisches Denken und die Verarbeitung von traumatischen Ereignissen. In: Steinhardt, K., Büttner, Ch. \& Müller, B. (Hrsg.): Kinder zwischen drei und sechs. Bildungsprozesse und Psychoanalytische Pädagogik im Vorschulalter. Jahrbuch für Psychoanalytische Pädagogik Bd. 15. Frankfurt, 39-56.

Camilleri, C. (1990): Stratégies identitaires. Paris.

Erikson, E. H. (1959): Identität und Lebenszyklus. Berlin. 


\section{Lehren und Lernen im Kontext von Flucht und Neubeginn}

Freud, A. \& Burlingham, D. (1987): Kriegskinder: Berichte aus den Kriegskinderheimen "Hampstead nurseries". Bd. 2. 1939-1945. Frankfurt.

Gerspach, M. (2018): Psychodynamisches Verstehen in der Sonderpädagogik. Stuttgart.

Kothe-Meyer, I. (1999): Ich-Funktionen und Ich-Identität im Wechsel von Sprache und kulturellem Raum. In: Pedrina, F., Saller, V., Weiss, R. \& Würgler, W. (Hrsg.): Kultur, Migration, Psychoanalyse. Stuttgart, 71-99.

Ottomayer, K. (2013): Die Behandlung der Opfer: Über unseren Umgang mit dem Trauma der Flüchtlinge und Verfolgten. Stuttgart.

Pedrina, F. (1999): Identitätsentwicklung in einer Welt der Begegnung. In: Pedrina, F., Saller, V., Weiss, R. \& Würgler, W. (Hrsg.): Kultur, Migration, Psychoanalyse. Stuttgart, 45-71.

Rüdi, J. (2001): Das Kind mit unkontrollierten Verhaltensweisen. In: Winkel, R. (Hrsg.): Schwierige Kinder - problematische Schüler. Batmannsweiler, 267-293.

Salzberger-Wittenberg, I. (1997): Über das Beginnen. In: Salzberger-Wittenberg, I., HenryWilliams, G. \& Osborne, E. (Hrsg.): Die Pädagogik der Gefühle. Wien, 17-38.

Streeck-Fischer, A. (2014): Trauma und Entwicklung: Adoleszenz - frühe Traumatisierungen und ihre Folgen. Stuttgart.

Terr, L. (1995): Schreckliches Vergessen, heilsames Erinnern. Traumatische Erfahrungen drängen ans Licht. Hamburg.

Weiss, W. (2014): Traumasensible Familienhilfe - ein Beitrag zur Psychosozialen Traumatologie. In: Gahleitner, S., Hensel, T., Baierl, M., Kühn, M. \& Schmid, M. (Hrsg.): Traumapädagogik in psychosozialen Handlungsfeldern. Basel, 142-150. 